\title{
IDETC2019-97872
}

\section{ADDITIVE MANUFACTURING OF A BISTABLE MECHANISM USING FUSED DEPOSITION MODELING: EXPERIMENTAL AND THEORETICAL CHARACTERIZATION}

\author{
Mouna Ben Salem* \\ Guillaume Aiche \\ Yassine Haddab \\ Laboratory of Informatics, Robotics \\ and Microelectronics \\ University of Montpellier \\ Montpellier, 34000 France \\ Email: bensalem, aiche, haddab@lirmm.fr
}

\author{
Lennart Rubbert \\ Pierre Renaud \\ The Engineering Science, Computer \\ Science and Imaging Laboratory \\ University of Strasbourg \\ Strasbourg, 67000 France \\ Email: lennart.rubbert, \\ pierre.renaud@insa-strasbourg.fr
}

\begin{abstract}
Bistable mechanisms can be used for performing specific functions such as locking or negative stiffness generation. These compliant structures are then of interest at different scales, with different corresponding manufacturing technologies. One of them is additive manufacturing, which is interesting for the integration of such structures. Although this technology has undergone a revolution with the development of high-accuracy machines, the manufacturing of small-sized compliant structures is still quite a challenge especially for bistable mechanisms, which was not yet finely characterized. This is the focus of this paper, with presentation of an experimental and analytical confrontation in the case of Fused Deposition Modeling (FDM).
\end{abstract}

\section{INTRODUCTION}

Additive Manufacturing (AM) also known as 3D printing is a fundamentally different technology in comparison to the more traditional machining processes. It consists in building parts based on the principle of turning a CAD model into a physical 3D object by adding several layers of material [1].

This technology has several benefits. First, it facilitates the fab- rication of complex shapes that cannot be produced by conventional methods, for instance the manufacturing of compliant structures at different scales. It offers the use of a quite large range of materials. 3D printed parts can now exhibit high heat resistance, high stiffness and even be biocompatible. AM also allows low-cost and fast prototyping which can highly accelerate the design cycle [1] [9]. Although the benefits of this manufacturing process are very attractive, it still has its own limitations. Some of them are related to the process, other can be related to the characteristics of the printer being used. The layer-per-layer production of parts makes them weaker than parts made by traditional processes. The dimensional accuracy, the structure of the printed parts and the integrity of the layers depends on the process and the machine settings [1] [9] [16].

In this paper, we study the impact of $\mathrm{AM}$, more precisely material extrusion Fused Deposition Modeling (FDM), which is one of the seven families of AM (according to the standard ASTM F2792) [2], on bistable structures [6]. The bistable behavior defines this kind of structure and has been used in several applications at different scales [3] [4]. One of the applications is a medical one that we are currently developing in which the bistable mechanism offers a locking property to manage sampling of intestinal liquid in the human body [4]. The bistable mechanism is 


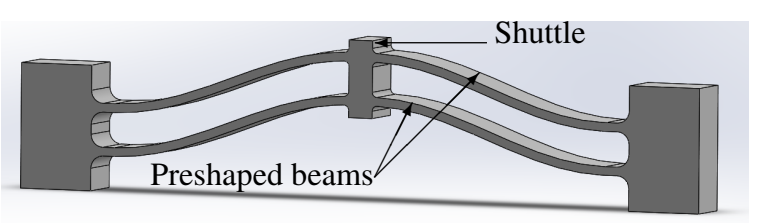

FIGURE 1: Representation of a double-beam based bistable mechanism in its first stable position

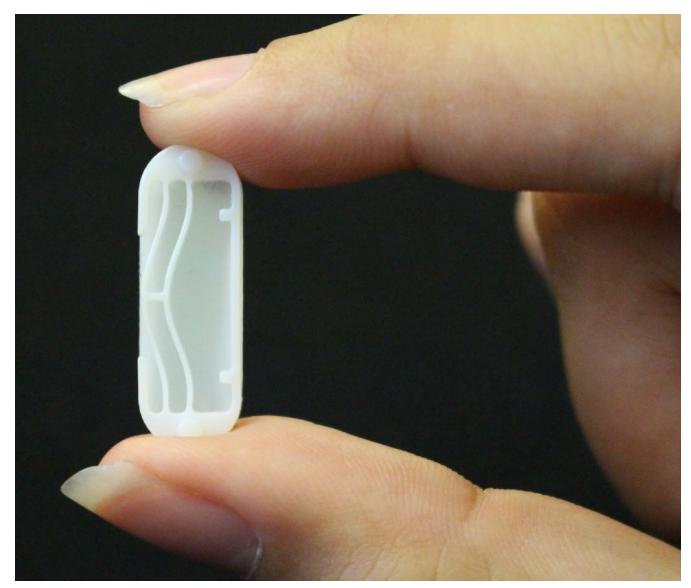

FIGURE 2: 3D printed miniaturized bistable mechanism

then manufactured in a so-called miniature version compared to the first bistable mechanism considered in this paper (as shown in Figure 2).

The bistable mechanism considered here is composed of the superposition of two preshaped curved beams that follows an initial shape when manufactured as shown in Figure 1. They are connected together with a shuttle (see Figure (1) to avoid rotation of the center of the beams during their movement from the first stable position to the second [7]. Given its size, 3D printing of the structure becomes challenging in term of dimensional accuracy and bistability assurance. Therefore a study is carried out to investigate the practical feasibility of FDM-based bistable mechanisms and the adjustment of the manufacturing process at two different scales. This work allows also to differentiate the scalerelated effects from the ones related to the AM technology used. In Section 2, the design and the initial theoretical characterization of the bistable mechanism are introduced along with the mechanisms we consider. In section 3 , the manufacturing technology and the manufactured parts are presented along with the results of the dimensional accuracy of the printed structures. Section 4 is dedicated to the experimental characterization of the bistable behavior considering the influence of AM and the determination of the Young's modulus after manufacturing. Finally conclusions

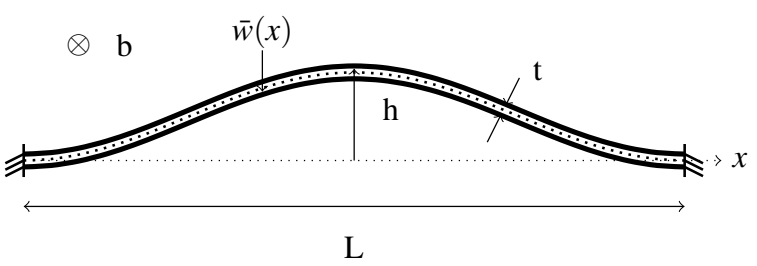

FIGURE 3: Preshaped curved beam at the initial position

and further work are discussed in section 5

\section{Modeling of a bistable mechanism \\ 2.1 Analytical modeling of a bistable mechanism}

The bistable mechanism used in this paper is composed of the superposition of two preshaped curved beams that have the characteristics shown in Fig 3 thickness $t$, depth $b$, length $L$, initial beam shape $\bar{w}(x)$ and initial height $h$ [7] [5]. This configuration defines the bistability of the structure. These beams are manufactured according to the shape parameterized by the equation (1) and they are connected to each other in the middle as represented in Fig 1 without affecting the beam length and shape.

$$
\bar{w}(x)=\frac{h}{2}\left[1-\cos \left(2 \pi \frac{x}{L}\right)\right]
$$

To ensure that the mechanism has a bistable behavior, the ratio between the initial height $h$ and the thickness $t$ must exceed 2.31 [5]. Once this behavior exists, it can be characterized by the evolution of the applied force $f$ and the displacement $d$ of its center as shown in Fig 4 . The extrema of this curve $f_{\text {top }}$ and $f_{\text {bot }}$ are the forces needed to be provided to trigger the structure and switch between the two bistable positions. The value of these forces can be calculated, along with the corresponding displacements, using the following expressions [5]:

$$
\begin{gathered}
d_{\text {top }}=h\left(\frac{28}{27}-\frac{2 \pi}{3} \sqrt{\frac{1}{6}+\frac{16}{81 \pi^{2}}-\frac{1}{Q^{2}}}\right) \\
d_{\text {bot }}=h\left(\frac{28}{27}+\frac{2 \pi}{3} \sqrt{\frac{1}{6}+\frac{16}{81 \pi^{2}}-\frac{1}{Q^{2}}}\right) \\
d_{\text {end }}=h\left(\frac{3}{2}+\sqrt{\frac{1}{4}-\frac{4}{3 Q^{2}}}\right) \quad, \quad d_{\text {mid }}=1.33 h
\end{gathered}
$$




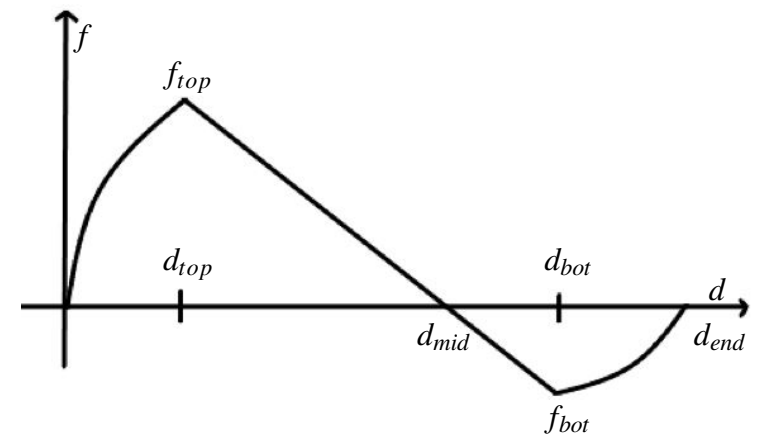

FIGURE 4: Force-Displacement curve of a bistable mechanism

TABLE 1: Dimensions of the large bistable mechanism

\begin{tabular}{|c||c|c|c|c|}
\hline Geometric parameter & $\mathrm{L}$ & $\mathrm{t}$ & $\mathrm{b}$ & $\mathrm{h}$ \\
\hline Value & $187 \mathrm{~mm}$ & $2 \mathrm{~mm}$ & $5 \mathrm{~mm}$ & $14 \mathrm{~mm}$ \\
\hline$f_{\text {top }}=64 \pi^{2} \frac{E I h}{L^{3}}\left(\frac{4}{3}-\frac{d_{\text {top }}}{h}\right), \quad f_{\text {bot }}=64 \pi^{2} \frac{E I h}{L^{3}}\left(\frac{4}{3}-\frac{d_{\text {bot }}}{h}\right)$
\end{tabular}

with $E$ the Young's modulus of the material, $I$ the quadratic moment and $Q=\frac{h}{t}$.

These expressions are obtained from an analytical modeling of the buckling phenomenon [6]. Comparison between analytical and experimental results for bistable mechanisms are presented in [5] using these expressions.

Based on this analytical model and the specifications of the printer that is used to manufacture the bistable structures (Fortus $400 \mathrm{mc}$, Stratasys Ltd, USA), two scales of dimensions are identified. The first scale allows to manufacture large bistable mechanisms with dimensions that allows the deposition of several layers of materials. These dimensions are illustrated in Table 1

As for the second scale, it allows to manufacture small-sized bistable mechanism, with in this case only one single deposited layer. Its dimensions are given in Table 2. These dimensions are small enough to fit the medical application [4] and get the bistability of the mechanism. The two bistable mechanisms are selected to get very similar bistability factor $Q: Q_{\text {large }}=7$ and $Q_{\text {minimized }}=6.8$.

\subsection{Initial Finite Elements model}

As an initial Finite Element (FE) model, a 2D modeling of the bistable mechanism is used to simulate the bistable behavior of the mechanisms and to determine the force-displacement
TABLE 2: Dimensions of the miniaturized bistable mechanism

\begin{tabular}{|c||c|c|c|c|}
\hline Geometric parameter & $\mathrm{L}$ & $\mathrm{t}$ & $\mathrm{b}$ & $\mathrm{h}$ \\
\hline Value & $20 \mathrm{~mm}$ & $0.35 \mathrm{~mm}$ & $2 \mathrm{~mm}$ & $2.4 \mathrm{~mm}$ \\
\hline
\end{tabular}

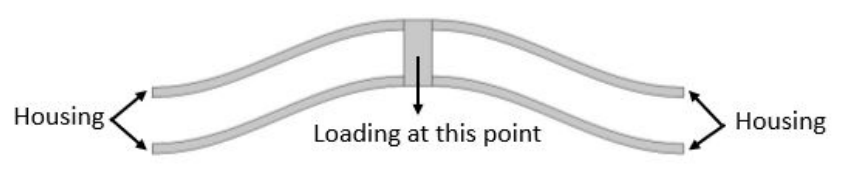

FIGURE 5: 2D model of the bistable mechanism

curve using Comsol software (COMSOL Inc.). The bistable mechanism is composed of two beams that follow the equation (1) and which are connected to each other by a central block as represented in Figure 5. To respect the boundary conditions on which the analytical model is based, a housing is built connecting both ends of the mechanism. As for the mesh used for the FE simulation, the size of the maximum element is $0.02 \mathrm{~mm}$, the size of the minimum element is $410^{-5} \mathrm{~mm}$ and the number of elements is 1350. The force and displacement are calculated at the middle of the central block.

This FE model is used to assess the behavior of the two bistable mechanisms predicted by the analytical model. For the simulations, polycarbonate is considered as it is the material used to manufacture the structure. It has a Young's modulus equal to $1944 \mathrm{MPa}$. The comparison between the force-displacement curves given by the analytical model and the FE simulations are given respectively in Figure 6 and Figure 7 .

One can notice, for the large bistable mechanism, the good agreement between the FE curve and the theoretical one. On the other hand, for the miniaturized mechanism, a difference of $21 \%$ can be observed for the maximum forces and $38 \%$ for minimum forces. The analytical model is determined on the basis of different assumptions. One of these hypotheses is $L \gg h \gg t$ so the length of these beams is chosen to be long enough to be able to consider a small displacement at the borders with respect to the length [5]. This hypothesis is not met for miniaturized bistable mechanisms, which explains the observed discrepancy.

Given the important difference between the two results, the analytical model is considered invalid for the miniaturized bistable in terms of results for the triggering force values. Therefore, it is important to go through FE modeling for the initial characterization of small-sized bistable mechanism. 


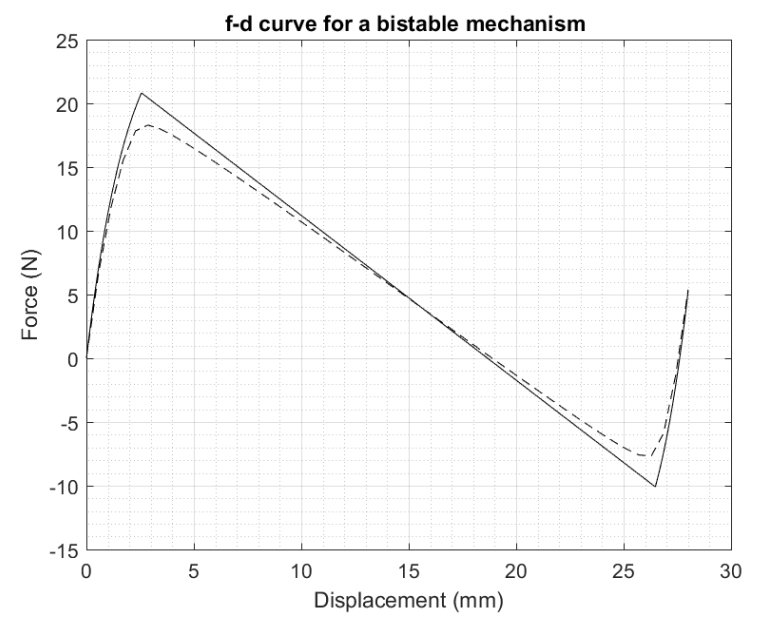

FIGURE 6: Force-Displacement curves of the large bistable mechanism: analytical model presented in continuous line and FE simulation in dashed line

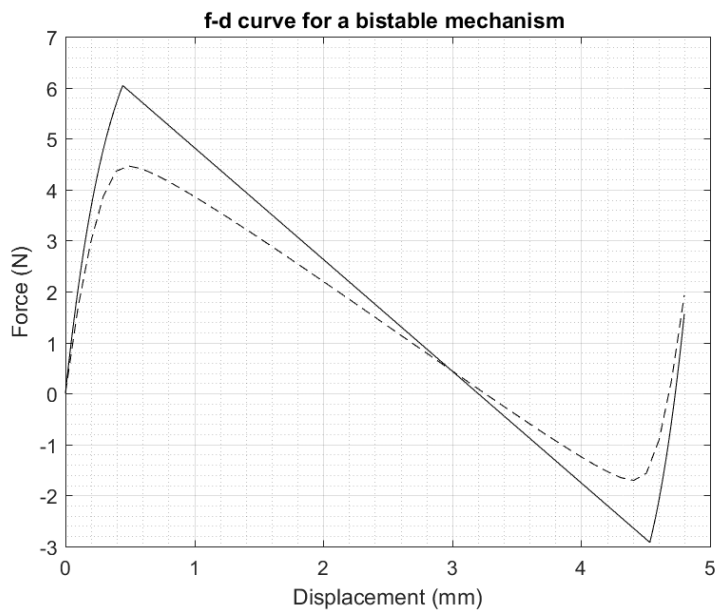

FIGURE 7: Force-Displacement curves of the miniaturized bistable mechanism: analytical model presented in continuous line and FE simulation in dashed line

\section{Additive manufacturing of the bistable mechanism 3.1 Fused Deposition Modeling: principle and main parameters}

Fused Deposition modeling (FDM) is a technology developed by Stratasys Ltd. It consists in the deposit of a fused filament in successive layers through a nozzle. Therefore the final part consists of a stack of thin layers [16]. There are many process parameters in this technology. Some are machine parameters such as the nozzle diameter and the filament diameter. Oth- ers can be considered as operational parameters such as the layer thickness, the head speed, the fill pattern and the part orientation. There are also parameters related to the material used. The impact of these parameters on the quality of the printed parts has been the subject of several studies. For instance, the influence of parts orientation on the quality of printed parts has been studied in [19] and fill pattern in [20] and [21].

For parts of small size, several factors can influence their performances which can be the dimension accuracy [11] [12], the surface roughness [13] [14], the mechanical properties [15] or the presence of underfill/overfill areas [10]). For instance Santhakumar in [8] showed that the optimum parameters for maximum impact strength are $0.254 \mathrm{~mm}$ for the layer thickness and $30^{\circ}$ for the build orientation. In [17], the deposit filament direction is discussed according to the removal direction of the parts from the tray of the machine after manufacturing because this manipulation can lead to a plastic behavior of the manufactured parts. In [18] the dimensional accuracy and the Young's modulus are investigated according to the building direction. The results show a positive error between the theoretical parameters and the printed one, which means that the printer tends to create larger and bigger parts. Based on these studies, the manufacturing parameters (the layer thickness and the nozzle used) are identified. The bistable structures are manufactured using an industrial printer (Fortus $400 \mathrm{mc}$, Stratasys Ltd). The nozzle used to manufacture the mechanisms allows to deposit a minimal layer thickness equal to $0.25 \mathrm{~mm}$. Therefore, for the large bistable mechanism, the beam will consist of a superposition of 8 layers (the layers are deposited in the form of closed contour) in the y direction and 29 layers in the $\mathrm{z}$ direction as one can see in Figure 8 and Figure 9

The deposited layers are fused together to build the bistable mechanism but it was observed experimentally that some underfill occurs as shown in Figure 8

Figure 9 shows a part of the beam of the large bistable mechanism manufactured with FDM technology, with a $\times 30$ zoom. The part that connects the beam with its frame, where the boundary conditions must be respected, is underfilled. The dimensions of this underfill are given in Figure 10

As for the miniaturized bistable mechanism, the nozzle deposits a single layer in the y direction that has a thickness equal to the total thickness of the mechanism which is in this case $0.35 \mathrm{~mm}$ as shown in Figure 11 .

Considering these manufacturing parameters, the dimensional accuracy of the manufactured parts will be studied in the following, along with an experimental determination of the Young's modulus as the value of the latter changes after manufacturing and can therefore impact the value of the snapping forces $f_{\text {top }}$ and $f_{b o t}$ of bistable mechanisms. 


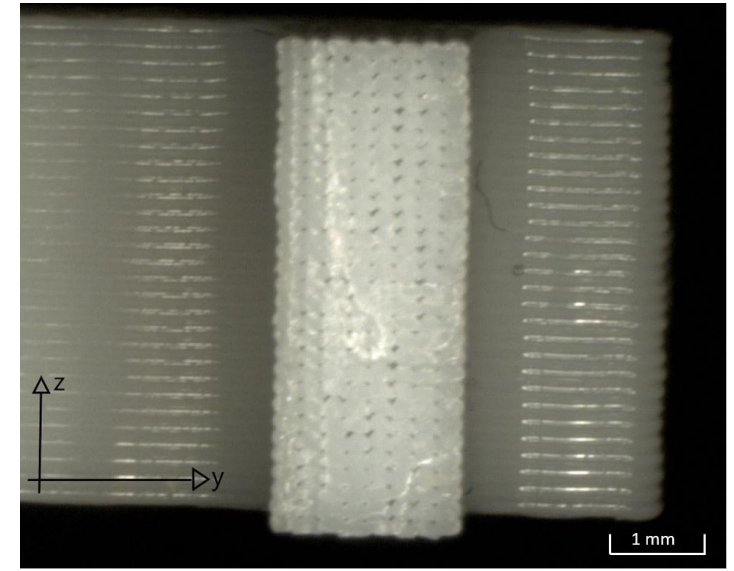

FIGURE 8: $\times 30$ zoom of a cut of the manufactured beam

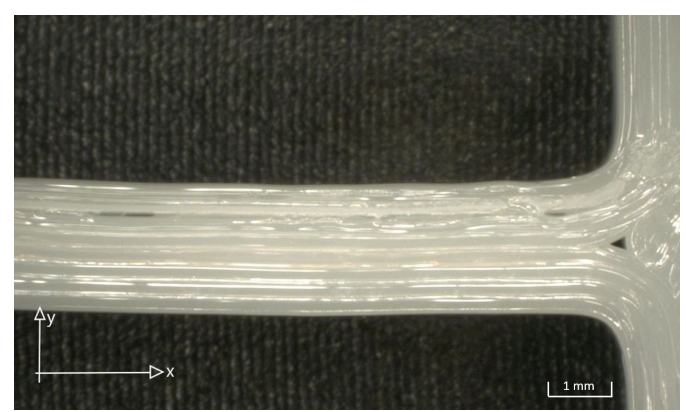

FIGURE 9: $\times 30$ zoom of a beam from the large bistable mechanism

\subsection{Dimensional accuracy of the printed parts}

To carry out the experimental characterization of the bistable behavior, a set of 10 bistable mechanisms are manufactured for each scale. After manufacturing, the dimensions of the mechanisms are measured to determine the dimensional accuracy. The dimensions $h$ and $L$ are the most important dimensions to measure since they can influence the force-displacement evolution. The dimensions are measured by vision with a 16 MP camera, which allows to assess the dimensions with a $0.19 \mu \mathrm{m}$ accuracy. The measurements and the experimental characterization are done 24 hours after manufacturing in order to let the mechanism cool off and to avoid any kind of twisting of the parts when they are removed from the tray. The same steps are repeated a week later to evaluate the aging of the parts and its influence on their bistable behavior. The one-week delay is somehow arbitrarily chosen, even though this could be the minimal delay between production and use in the intended medical context. During one week, six switching cycles were preformed for each mechanism. The post-manufacturing dimensions of the 10 printed samples

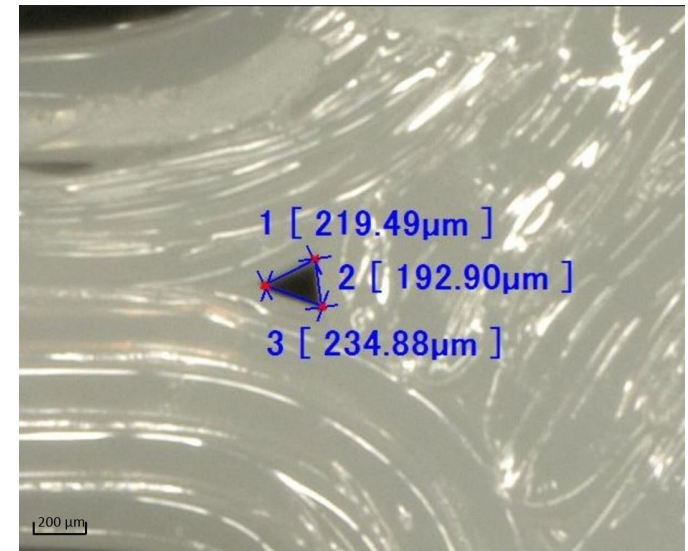

FIGURE 10: Dimensions of the underfill in the large bistable mechanism

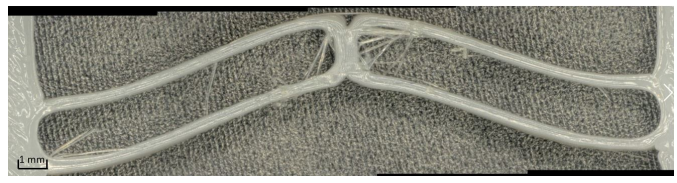

FIGURE 11: $\times 50$ zoom of the miniaturized bistable mechanism

are presented in Table 3 for the large bistable mechanism and in Table 4 for the miniaturized one.

\section{Experimental evaluation}

\subsection{Experimental setup}

In order to characterize the bistable mechanism, a dedicated setup was built. It is made up of a laser-based displacement sensor (LK-H152, Keyence) that has a measuring range of $80 \mathrm{~mm}$ with a resolution of $0.25 \mu \mathrm{m}$, a force sensor (FK50, Sauter) with a maximum force of $50 \mathrm{~N}$ and an accuracy of $0.02 \mathrm{~N}$ and a mounting bracket to fix the bistable mechanism and ensure the boundary conditions. An additional cover is added and fixed with screws to prevent the movement of the mechanism during the experiment. The initial positioning is achieved to guarantee that no axial load is imposed by the screws.

A force is applied to the bistable mechanism by moving the linear table on which the force sensor is mounted and the corresponding displacement is measured using the laser sensor. Once the positive forces are determined, the bistable mechanism is reversed to determine the negative forces.

The same experimental setup is used to characterize the bistable behavior of the miniaturized mechanisms. Only the mounting bracket is adapted to the dimensions of the structures as shown in Figure 13 Considering the dimensions of the central block 
TABLE 3: Post-manufacturing dimensions of the large bistable mechanism during one week

\begin{tabular}{|c|l|l|l|l|}
\hline \multirow{2}{*}{ Sample } & \multicolumn{2}{|c|}{ After one day } & \multicolumn{2}{c|}{ After seven days } \\
\cline { 2 - 5 } & $L$ & $h$ & $L$ & $h$ \\
\hline 1 & $187.5 \mathrm{~mm}$ & $14.1 \mathrm{~mm}$ & $187.6 \mathrm{~mm}$ & $13.8 \mathrm{~mm}$ \\
\hline 2 & $187.3 \mathrm{~mm}$ & $13.8 \mathrm{~mm}$ & $187.5 \mathrm{~mm}$ & $13.6 \mathrm{~mm}$ \\
\hline 3 & $187 \mathrm{~mm}$ & $14.1 \mathrm{~mm}$ & $187.4 \mathrm{~mm}$ & $13.7 \mathrm{~mm}$ \\
\hline 4 & $187.1 \mathrm{~mm}$ & $14.3 \mathrm{~mm}$ & Broken & Broken \\
\hline 5 & $187.2 \mathrm{~mm}$ & $13.9 \mathrm{~mm}$ & $187.6 \mathrm{~mm}$ & $13.9 \mathrm{~mm}$ \\
\hline 6 & $187.1 \mathrm{~mm}$ & $13.7 \mathrm{~mm}$ & $187.3 \mathrm{~mm}$ & $13.9 \mathrm{~mm}$ \\
\hline 7 & $187 \mathrm{~mm}$ & $14.1 \mathrm{~mm}$ & $187.7 \mathrm{~mm}$ & $14 \mathrm{~mm}$ \\
\hline 8 & $187.2 \mathrm{~mm}$ & $14 \mathrm{~mm}$ & $187.6 \mathrm{~mm}$ & $14 \mathrm{~mm}$ \\
\hline 9 & $187.1 \mathrm{~mm}$ & $14.5 \mathrm{~mm}$ & $187.2 \mathrm{~mm}$ & $14.3 \mathrm{~mm}$ \\
\hline 10 & $187.3 \mathrm{~mm}$ & $14.5 \mathrm{~mm}$ & $187.6 \mathrm{~mm}$ & $14.2 \mathrm{~mm}$ \\
\hline
\end{tabular}

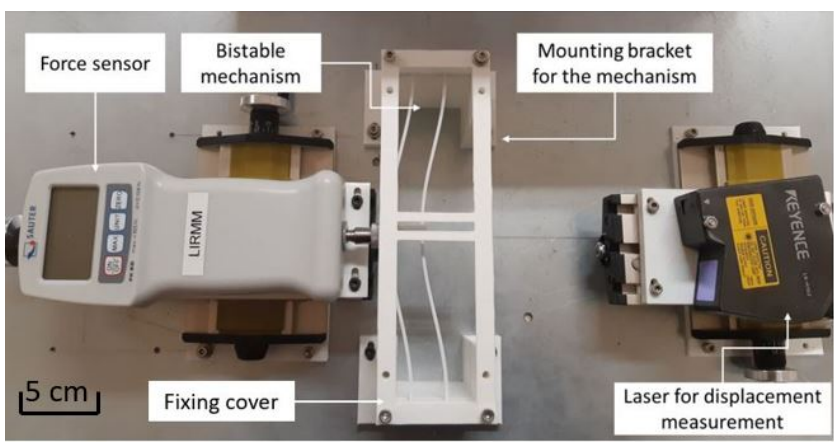

FIGURE 12: Experimental setup for the characterization of the large bistable mechanism

of the miniaturized mechanisms, a mounting bracket (as shown in Figure 13 is used to ensure that the mechanism rotation is blocked before starting the tests.

\subsection{Experimental determination of Young's modulus}

To determine the Young's modulus of material after manufacturing, a test beam is produced with the printing parameters described previously. The experimental setup is slightly modified (Figure 14) to allow the determination of the force-displacement
TABLE 4: Post-manufacturing dimensions of the miniaturized bistable mechanism during one week

\begin{tabular}{|c|l|l|l|l|}
\hline \multirow{2}{*}{ Sample } & \multicolumn{2}{|c|}{ After one day } & \multicolumn{2}{c|}{ After seven days } \\
\cline { 2 - 5 } & $L$ & $h$ & $L$ & $h$ \\
\hline 1 & $21.5 \mathrm{~mm}$ & $2.3 \mathrm{~mm}$ & $22 \mathrm{~mm}$ & $2.2 \mathrm{~mm}$ \\
\hline 2 & $21.8 \mathrm{~mm}$ & $2.3 \mathrm{~mm}$ & $22 \mathrm{~mm}$ & $2.2 \mathrm{~mm}$ \\
\hline 3 & $21.5 \mathrm{~mm}$ & $2.4 \mathrm{~mm}$ & $21.5 \mathrm{~mm}$ & $2.3 \mathrm{~mm}$ \\
\hline 4 & $21.4 \mathrm{~mm}$ & $2.5 \mathrm{~mm}$ & $22.5 \mathrm{~mm}$ & $2.5 \mathrm{~mm}$ \\
\hline 5 & $21.6 \mathrm{~mm}$ & $2.3 \mathrm{~mm}$ & $21.7 \mathrm{~mm}$ & $2.1 \mathrm{~mm}$ \\
\hline 6 & $21.4 \mathrm{~mm}$ & $2.5 \mathrm{~mm}$ & $21.5 \mathrm{~mm}$ & $2.5 \mathrm{~mm}$ \\
\hline 7 & $21.3 \mathrm{~mm}$ & $2.4 \mathrm{~mm}$ & $22 \mathrm{~mm}$ & $2.3 \mathrm{~mm}$ \\
\hline 8 & $21.7 \mathrm{~mm}$ & $2.4 \mathrm{~mm}$ & $21.8 \mathrm{~mm}$ & $2.3 \mathrm{~mm}$ \\
\hline 9 & $21.2 \mathrm{~mm}$ & $2.2 \mathrm{~mm}$ & $22.1 \mathrm{~mm}$ & $2.2 \mathrm{~mm}$ \\
\hline 10 & $21.3 \mathrm{~mm}$ & $2.4 \mathrm{~mm}$ & $22 \mathrm{~mm}$ & $2.3 \mathrm{~mm}$ \\
\hline
\end{tabular}

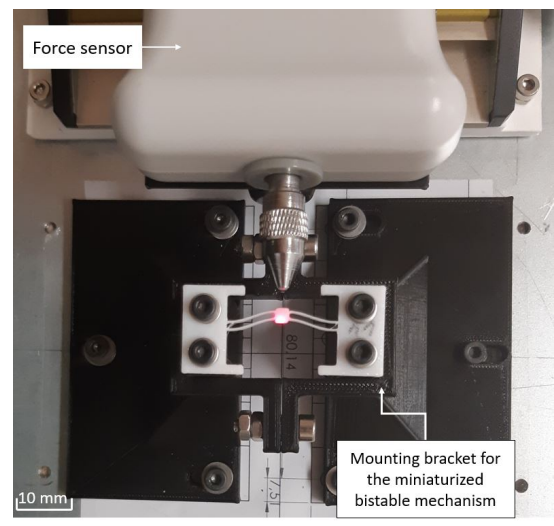

FIGURE 13: Mounting bracket used to lock the miniaturized bistable mechanisms

characteristic. The Young's modulus is determined from beam bending, using the relationship issued from the Euler-Bernoulli theory:

$$
v=\frac{F L^{3}}{3 E I}
$$

where $F$ is the force applied on the test beam at its tip, $v$ the corresponding displacement, $E$ the Young's modulus to determine, 


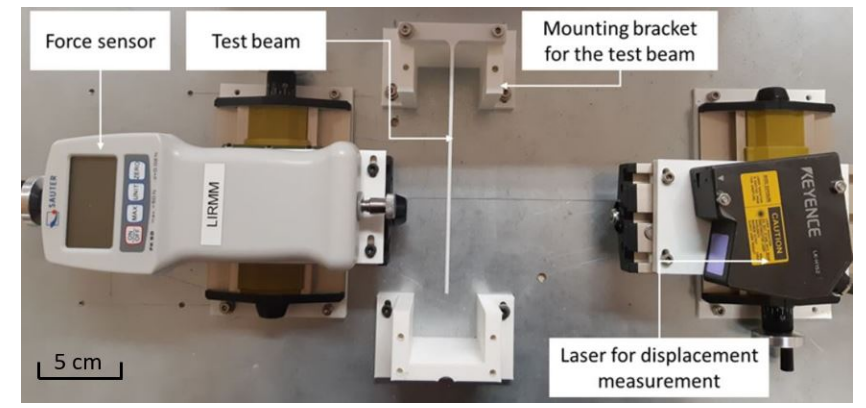

FIGURE 14: Setup for the determination of Young's modulus after manufacturing

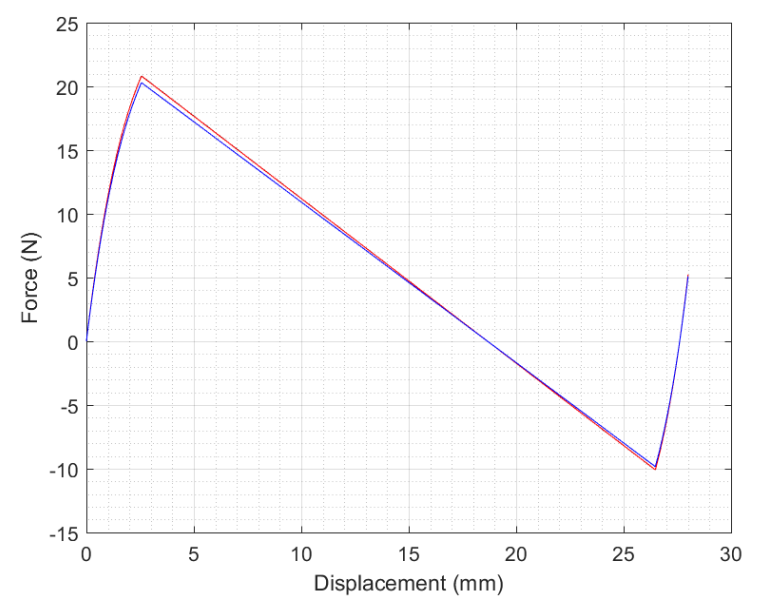

FIGURE 15: Force-Displacement of the large bistable mechanism, in red using the Young's modulus measured $24 \mathrm{~h}$ after manufacturing is used and in blue using the one measured one week later

$I$ the quadratic moment of the cross section and $L$ the length of the test beam.

The experiments are repeated a week later to evaluate the influence of the aging of the parts on the Young's modulus. 24 hours after production, the average value of the Young's modulus is $1943 \mathrm{MPa}$, to compare to the manufacturer value of $1944 \mathrm{MPa}$. One week later, the average value has decreased to $1895 \mathrm{MPa}$. In order to assess the impact of such variations, the identified values are integrated in the force-displacement evolution of the large bistable mechanism. The results of this comparison are represented in Figure 15

\subsection{Experimental characterization of the bistable mechanisms}

The bistable behavior of each printed mechanism is characterized by experiments which consists in the determination of the force-displacement behavior for each sample with an interval of one week between the two tests. These results are then compared to the results obtained with the analytical model and the values of the Young's modulus identified from the specific experiments. The results of the large bistable mechanisms are presented in Figure 17 and those of the miniaturized mechanisms are presented in Figure 18

Figure 17 shows sudden jumps (like the one in Figure $17 \mathrm{~b}$ Figure $17 \mathrm{c}$ and Figure $17 \mathrm{i}$ in the Force-Displacement curves, especially between the maximum and the minimum forces. These jumps might be linked to the unexpected shapes that the bistable mechanism takes as given in Figure 16

After manufacturing, the Young's modulus decreases. Since the expressions of the forces are proportional to $E$, it has a major impact on the error on estimating the forces rather than the one estimating the equilibrium and zero stiffness positions.
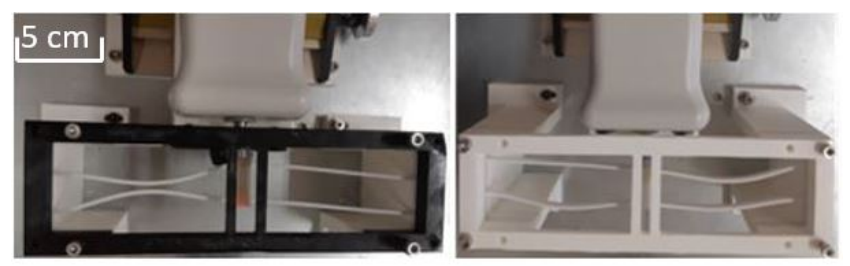

FIGURE 16: Unexpected shapes of the bistable mechanism going from its first stable position to the second one

\section{Discussion and conclusion}

First, dimensional measurements for the length $L$ and the initial height $h$ were made to determine the dimensional accuracy of the manufactured mechanisms 24 hours after manufacturing and one week after. For the large bistable mechanisms, the length of the structure is equal on average to $187_{-0}^{+0.5} \mathrm{~mm}$ and the initial height is equal on average to $14_{-0.3}^{+0.5} \mathrm{~mm}$. One week later and after several cycles of use, the length of the structures increases by up to $0.37 \%$ and the initial height decreases by $2.84 \% \mathrm{~mm}$. For the miniaturized bistable mechanisms, the length of the structure is equal on average to $20_{+1.2}^{+1.8} \mathrm{~mm}$ and the initial height is equal on average to $2.4_{-0.2}^{+0.1} \mathrm{~mm}$. One week later and after several cycles of use, the length of the structures increases by up to $5.14 \%$ and the initial height decreases by $8.7 \%$. 
We can then notice that after using the bistable mechanisms, and an aging of one week, the structures present a relaxation effect, which results in an increase of the length with simultaneously a decrease of the initial height. As for the bistability, it is ensured for the two bistable mechanisms and no plastic deformation is however remarked.

The Young's modulus was determined after manufacturing. The results show that the value of the latter is $0.05 \%$ smaller than the theoretical value and within one week, its value decreases by $2.5 \%$. Finally, the comparison between the theoretical and experimental results, with a week-long aging of the structures, shows that for the large bistable mechanisms the values of the maximum forces are in agreement with the theoretical values, which is not the case for the minimum forces. For the displacements, one can notice a difference in the final position of the mechanism which is due to the relaxation of the parts. During the experiments, only one mechanism has been broken underfill level shown in section 3. The results concerning the miniaturized bistable mechanisms indicate a significant difference between the theoretical and experimental curves. The experimental results are closer to the FE results than the theoretical ones. The values of minimum forces are remarkably small for the miniaturized structures manufactured with FDM process as it does not exceed a maximum value of $0.38 \mathrm{~N}$. During the experiments, two mechanisms have been broken at the location of the central block of structures.

As a summary, we have investigated in this paper the production of bistable mechanisms with FDM. A geometry of bistable mechanism composed of double precurved beams was considered. Two manufacturing scales have been assessed in order to compare between the analytical results and the experimental results of the bistable behavior characterization, and identify the influence of the manufacturing process for each scale. For the large version of mechanisms, relaxation effect is noticeable. Bistability is however always obtained, with a prediction accuracy of snapping forces using an analytical model which seems admissible. For the small size of mechanisms, the impact of FDM process is much stronger. Snapping forces are considerably lowered. If bistability is still obtained, such phenomenon requires a modeling taking into account FDM impact, which constitutes a perspective of this work.

\section{Acknowledgements}

This work was supported by the Investissements d'Avenir (Labex CAMI ANR-11-LABX-0004).

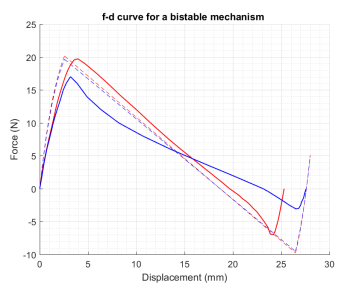

(a) Sample 1

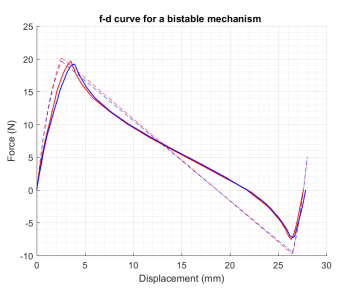

(c) Sample 3

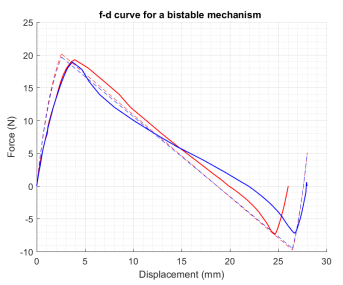

(e) Sample 6

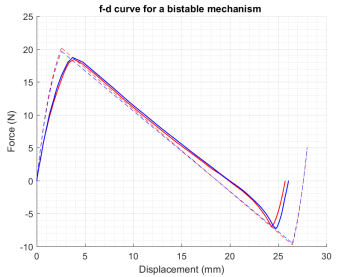

(g) Sample 8

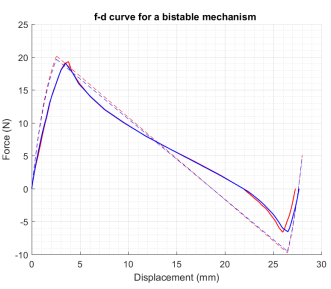

(b) Sample 2

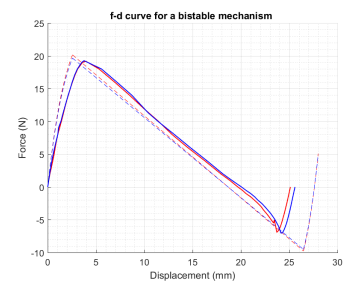

(d) Sample 5

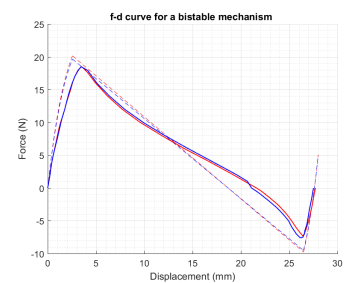

(f) Sample 7

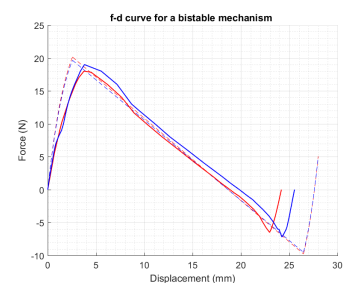

(h) Sample 9

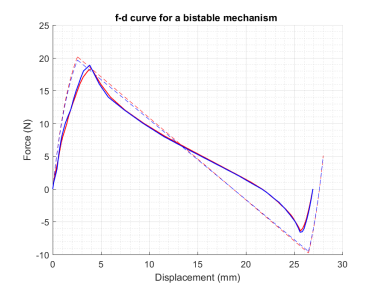

(i) Sample 10

FIGURE 17: Force-Displacement characteristics of large bistable mechanisms. In red, the characterization 24 hours after manufacturing and in blue the one one week later, in continuous line the experimental results and in dashed line the analytical results. 


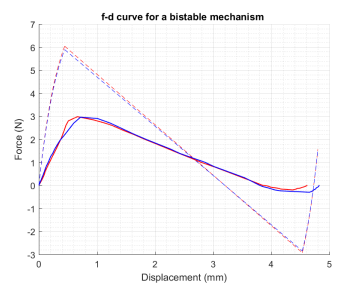

(a) Sample 2

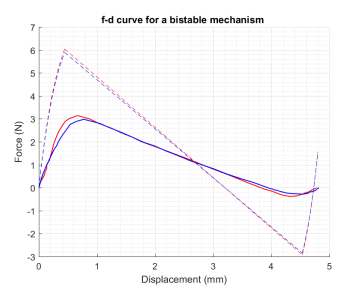

(c) Sample 4

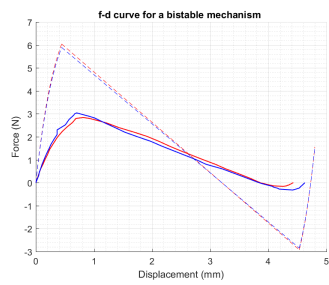

(e) Sample 6

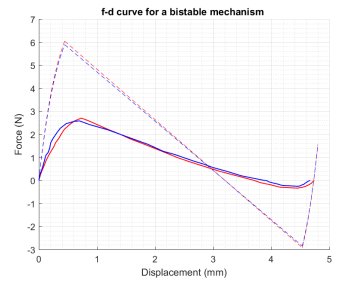

(g) Sample 9

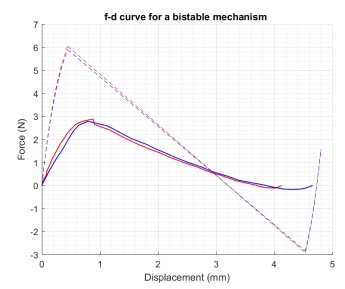

(b) Sample 3

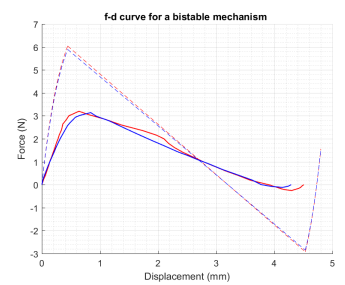

(d) Sample 5

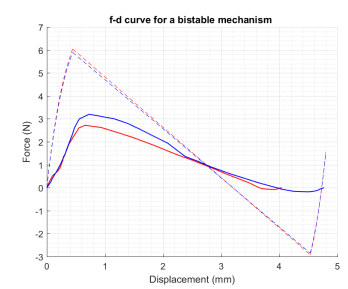

(f) Sample 7

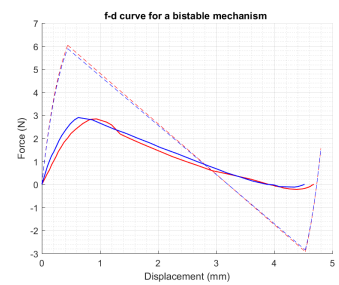

(h) Sample 10
FIGURE 18: Force-Displacement of the miniaturized bistable mechanisms. In red, the characterization 24 hours after manufacturing, in blue the one one week later, in continuous line the experimental results and in dashed line the analytical results.

\section{REFERENCES}

[1] DIZON, John Ryan C., ESPERA JR, Alejandro H., CHEN, Qiyi, et al. Mechanical characterization of 3D-printed polymers. Additive Manufacturing, 2018, vol. 20, p. 44-67.

[2] GIBSON, Ian, ROSEN, David et al. Additive manufacturing: rapid prototyping to direct digital manufacturing, 2010 Springer Science \& Business Media.

[3] HUSSEIN, Hussein, CHALVET, Vincent, LE MOAL, Patrice, et al. Design optimization of bistable modules electrothermally actuated for digital microrobotics. In : Ad- vanced Intelligent Mechatronics (AIM), 2014 IEEE/ASME International Conference on. IEEE, 2014. p. 1273-1278.

[4] BEN SALEM, Mouna, AICHE, Guillaume, RUBBERT, Lennart, et al. Design of a Microbiota Sampling Capsule using 3D-Printed Bistable Mechanism. In : 2018 40th Annual International Conference of the IEEE Engineering in Medicine and Biology Society (EMBC). IEEE, 2018. p. 4868-4871.

[5] HUSSEIN, Hussein. Contribution to Digital Microrobotics: Modeling, Design and Fabrication of Curved Beams, Ushaped Actuators and Multistable Microrobots. 2015 PhD thesis. Bourgogne Franche-Comté University.

[6] VANGBO, Mattias. An analytical analysis of a compressed bistable buckled beam. Sensors and Actuators A: Physical, 1998, vol. 69, no 3, p. 212-216.

[7] QIU, Jin. An electrothermally-actuated bistable MEMS relay for power applications. 2003. PhD thesis. Massachusetts Institute of Technology.

[8] SANTHAKUMAR, J., MAGGIRWAR, Rishabh, GOLLAPUDI, Srinivas, et al. Enhancing impact strength of fused deposition modeling built parts using polycarbonate material. Indian Journal of Science and Technology, 2016, vol. 9, no 34.

[9] HUANG, Tingting, WANG, Shanggang, et HE, Ketai. Quality control for fused deposition modeling based additive manufacturing: Current research and future trends. In : Reliability Systems Engineering (ICRSE), 2015 First International Conference on. IEEE, 2015. p. 1-6.

[10] FANG, Tong. Online image processing and defect detection in layered manufacturing using process signature. 2000 $\mathrm{PhD}$ thesis. The State University of New Jersey.

[11] M. JOHNSON, Wayne, ROWELL, Matthew, DEASON, Bill, et al. Comparative evaluation of an open-source FDM system. Rapid Prototyping Journal, 2014, vol. 20, no 3, p. 205-214.

[12] SONG, Suoyuan, WANG, Andi, HUANG, Qiang, et al. Shape deviation modeling for fused deposition modeling processes. In : Automation Science and Engineering (CASE), 2014 IEEE International Conference on. IEEE, 2014. p. 758-763.

[13] AHN, Daekeon, KWEON, Jin-Hwe, KWON, Soonman, et al. Representation of surface roughness in fused deposition modeling. Journal of Materials Processing Technology, 2009, vol. 209, no 15-16, p. 5593-5600.

[14] BOSCHETTO, Alberto, GIORDANO, V., et VENIALI, Francesco. Surface roughness prediction in fused deposition modelling by neural networks. The International Journal of Advanced Manufacturing Technology, 2013, vol. 67, no 9-12, p. 2727-2742.

[15] STEPHEN, A. O., DALGARNO, K. W., et MUNGUIA, J. Quality assurance and process monitoring of fused deposition modelling made parts. In : High value manufacturing: 
advanced research in virtual and rapid prototyping: proceedings of the 6th international conference on advanced research in virtual and rapid prototyping. 2013. p. 31-35.

[16] KIM, Chiyen, ESPALIN, David, CUARON, Alejandro, et al. A study to detect a material deposition status in fused deposition modeling technology. In : Advanced Intelligent Mechatronics (AIM), 2015 IEEE International Conference on. IEEE, 2015. p. 779-783.

[17] DOMINGO-ESPIN, Miquel, PUIGORIOL-FORCADA, Josep M., GARCIA-GRANADA, Andres-Amador, et al. Mechanical property characterization and simulation of fused deposition modeling Polycarbonate parts. Materials and Design, 2015, vol. 83, p. 670-677.

[18] QATTAWI, Ala, ALRAWI, Buraaq, GUZMAN, Arturo, et al. Experimental optimization of fused deposition modelling processing parameters: a design-for-manufacturing approach. Procedia Manufacturing, 2017, vol. 10, p. 791803.

[19] THRIMURThUlU, K. P. P. M., PANDEY, Pulak M., et REDDY, N. Venkata. Optimum part deposition orientation in fused deposition modeling. International Journal of Machine Tools and Manufacture, 2004, vol. 44, no 6, p. 585594.

[20] SINGAMNENI, Sarat, ROYCHOUDHURY, Asimava, DIEGEL, Olaf, et al. Modeling and evaluation of curved layer fused deposition. Journal of Materials Processing Technology, 2012, vol. 212, no 1, p. 27-35.

[21] LEE, Byoung Hun, ABDULLAH, J., et KHAN, Zahid A. Optimization of rapid prototyping parameters for production of flexible ABS object. Journal of materials processing technology, 2005, vol. 169, no 1, p. 54-61.

[22] TAUFIK, Mohammad et JAIN, Prashant K. Characterization, modeling and simulation of fused deposition modeling fabricated part surfaces. Surface Topography: Metrology and Properties, 2017, vol. 5, no 4, p. 045003. 